Article

\title{
In vitro Evaluation of Acyclovir/Chitosan Floating Systems
}

\section{Roberto Ruiz-Caro ${ }^{1}$ and María D. Veiga ${ }^{1,2, *}$}

1 Departamento de Farmacia y Tecnología Farmacéutica, Facultad de Farmacia, Universidad

Complutense de Madrid, Plaza Ramón y Cajal s/n, 28040-Madrid, Spain;

E-Mail: rruizcar@farm.ucm.es (R.R-C.)

2 Unidad de Biotransformaciones Industriales, Parque Científico de Madrid PTM, 28760-Tres Cantos, Madrid, Spain

* Author to whom correspondence should be addressed; E-Mail: mdveiga@farm.ucm.es; Tel.: +34-91-394-1741; Fax: +34-91-394-1736.

Received: 27 October 2010; in revised form: 24 November 2010 / Accepted: 25 November 2010 / Published: 6 December 2010

\begin{abstract}
Chitosan (CS) floating lyophilized formulations (L) for gastric drug delivery of acyclovir (ACV) have been developed. The freeze-dried formulations were obtained from acidic aqueous suspensions prepared with different ACV/CS ratios. No changes in ACV crystallinity were observed during X-ray diffraction powder studies as a consequence of the manufacturing process. Considering that fed and fasted states modified the intragastric $\mathrm{pH}$, swelling and in vitro dissolution studies were carried out in different acidic media $(0.1 \mathrm{M} \mathrm{HCl}$ and progressive $\mathrm{pH}$ medium) in order to understand the influence of these physiological states on ACV/CS formulations. Swelling behavior of the floating lyophilized formulations was dependent on CS and ACV proportions within L and on medium nature due to $\mathrm{pH}$ dependent $\mathrm{CS}$ solubility. Furthermore, no interactions between $\mathrm{ACV}$ and $\mathrm{CS}$ were detected in solid state according to the X-ray studies. In vitro dissolution of ACV from $\mathrm{L}$ was influenced by the swelling behavior. However, it is feasible to optimize the ACV/CS ratios to achieve a desired formulation that releases the total quantity of ACV at a specific time. Moreover, floatability was assessed by buoyancy tests. The results demonstrated that the freeze-drying process achieved effective floating systems capable of remaining within the stomach while the total amount of ACV is released from $\mathrm{L}$.
\end{abstract}


Keywords: Acyclovir; chitosan; floating freeze-dried formulations; swelling behavior; controlled release

\section{Introduction}

Nowadays, the excipients choice for drug formulation is focused on natural and biocompatible products. Chitosan (CS) is a biocompatible material obtained from an abundant natural polysaccharide like chitin by deacetylation processes [1]. Several excellent reviews have compiled different methods for obtaining a wide range of chitosans with different deacetylation degrees and molecular weights which affect some of their physico-chemical parameters [2-4]. This polysaccharide has been used for many different applications due to the fact that it exhibits a lot of biological actions, such as hypocholesterolemic, antimicrobial and wound healing properties [4-9]. Also, in pharmaceutical technology field CS has found applicability as a potential formulation excipient acting as a disintegrant, binder or tablet coating agent. This polymer presents a swelling ability when it is placed within an aqueous media [10], and mucoadhesivity in the oral cavity [11-13] and in the gastrointestinal tract [14]. Moreover, it has been widely used in solid oral formulations in order to obtain sustained release systems for hydrosoluble drugs [15-19]. Some authors have studied its ability to improve the dissolution of poorly water-soluble drugs [20-22]. Furthermore, CS crosslinks with tripolyphosphate or glutaraldehyde have been used as promising excipients to achieve colonic drug delivery [23]. Finally, CS citrate has been used to develop gels for vaginal administration of poorly permeable drugs [24].

Human herpes simplex virus type-1 (HSV-1) infects dermal epithelial and mucosal epithelial cells, causing lesions on the epithelium of the face, generally nose or lips [25,26]. Approximately, $80 \%$ of the adult population carries HSV-1, typically asymptomatic [26,27]. Primary infection usually occurs during childhood and, subsequent to the initial outbreak, the virus enters the peripheral nervous system, where it resides permanently in a latent state of infection. Many people never see an emergence of the disease from the latent stage of infection. Nevertheless, on average, 33\% of people infected with HSV-1 experience recurrences. Of these, 5\% have at least one episode per month, 34\% experience at least one episode every 2-11 months and 61\% have at least one episode per year [26,28]. Reactivation of the virus may occur as a consequence of fever, burns, exposure to ultraviolet light, emotional or physiological stress or hormonal changes [25,26,29]. In immunocompromized individuals, outbreaks occur with increasing frequency and are harder to control [30-32]. Most antiherpetic treatments are composed of a nucleoside analogue that inhibits herpes virus DNA polymerase, such as acyclovir (ACV) or valacyclovir [33]. Being soluble in the gastrointestinal media, ACV is absorbed paracellularly by passive diffusion from the upper region to the duodenum or jejunum regions $[34,35]$.

Some reports have suggested that the high variability of ACV effectiveness is related to saturable and dose dependent processes [36], thus its bioavailability is reduced to $15-30 \%$ [37]. In commercial available dosage forms, the amount of ACV absorbed is very low (10-20\%) because of the short residence time at the absorption site. As a result, approximately 50-60\% is excreted in the feces in its unabsorbed form. Considering its short half-life (about two hours) and its incomplete absorption, ACV must be taken orally five times per day or by intravenous administration every eight hours [38]. The 
inclusion of absorption-enhancing excipients in formulations can enhance ACV bioavailability. ACV chemical modifications have been performed to enhance its oral bioavailability $[39,40]$. Luengo et al. [41] studied the pharmacokinetics of different preparations of ACV within $\beta$-cyclodextrins. It was found that $\beta$-cyclodextrin had no significant effect on ACV oral bioavailability as $\beta$-cyclodextrins increase the solubility of lipophilic drugs, but not the permeability of hydrophilic drugs. Attia et al. developed ACV niosomes to improve its oral bioavailability with positive results in rabbits [42].

Advantages of time-controlled release oral dosage forms in optimizing therapeutic effectiveness and reducing side effects, are their capacity to minimize the fluctuation concentrations in plasma and to reside at the site of absorption for prolonged periods of time. These properties lead to an improvement in patient compliance due to a reduction in the administration frequency and therefore a decrease of the total dose administered while maintaining similar therapeutic effects. However, conventional controlled-release dosage forms offer only limited advantages for drugs that have an absorption window in the upper small intestine (e.g., ACV [43], furosemide [44] and riboflavin [45]). Once dosage forms are evacuated from the stomach, their passage through this region is rapid, thus limiting the extent of absorption at this site. In order to enhance the absorption and bioavailability of these drugs, the residence time of the controlled-release dosage forms in the upper gastrointestinal tract needs to be prolonged [46].

Gastroretentive dosage forms, like floating systems, have achieved some success in prolonging the gastric residence time [47]. Floating dosage forms are expected to remain buoyant on gastric media because they have a lower density than gastric fluids $\left(\sim 1.004 \mathrm{~g} / \mathrm{cm}^{-3}\right)$. Junyaprasert and Pornsuwannapha developed hollow microspheres of ACV that were capable of floating on gastric fluids providing longer residence time at the absorption site of ACV. This is because the loaded drug starts to dissolve slowly in the stomach and is absorbed into the systemic circulation in the narrow absorption window. Thus, these forms improved ACV absolute bioavailability, resulting in a reduction of the frequency of administration, which increases patient compliance [48].

Moreover, another advantage of floating systems is that they do not cause problems of mucus irritation. This side effect has been reported during the development of magnetic depot tablets as an alternative to obtain ACV oral sustained release [49].

Whitehead et al. demonstrate the feasibility of a freeze-drying process by $\gamma$-scintigraphy as an effective method to develop floating systems [50]. Further, Talukder and Fassihi developed pectin freeze-dried beads and the use of confocal laser microscopy revealed hollow spaces within the beads, which allowed them to remain buoyant for over 12 hours in gastric fluid [51].

After considering these studies, the aim of this research is to develop floating gastric formulations of $\mathrm{ACV} / \mathrm{CS}$ by freeze-drying processes, in order to obtain modulated drug release dosage forms in an attempt to increase ACV bioavailability.

\section{Results and Discussion}

Different ACV/CS systems (L) have been prepared by a freeze-drying process. Their compositions are shown in Table 1. In order to study interactions between CS and ACV, L and raw materials employed for their preparation have been characterized in solid state by X-ray diffraction powder. 
Furthermore, swelling and in vitro dissolution behavior of systems have been studied in order to determine their influence on ACV controlled release from L. Blank systems (B), which were prepared without ACV, have also been evaluated in swelling studies.

Table 1. Proportions of acyclovir (ACV) and chitosan (CS) in suspensions used to manufacture lyophilized systems.

\begin{tabular}{ccc}
\hline $\begin{array}{c}\text { Formulations } \\
\text { (F) }\end{array}$ & $\begin{array}{c}\text { Composition of suspensions before } \\
\text { freeze-drying process }(\mathrm{g} / 100 \mathrm{~mL})\end{array}$ \\
\hline L1 & ACV & CS \\
L2 & 0.5 & 1 \\
L3 & 0.5 & 2 \\
L4 & 0.5 & 3 \\
L5 & 0.5 & 4 \\
L6 & 0.5 & 5 \\
L7 & 2 & 1 \\
L8 & 2 & 2 \\
L9 & 2 & 3 \\
L10 & 2 & 4 \\
B1 & 2 & 5 \\
B2 & - & 1 \\
B3 & - & 2 \\
B4 & - & 3 \\
B5 & - & 4 \\
\hline
\end{tabular}

\subsection{X-ray Diffraction Analysis}

Figure 1 depicts X-ray diffraction powder patterns of raw ACV and CS and lyophilized systems in solid state. The diffraction pattern of ACV revealed its characteristic peaks at $7.0^{\circ}, 10.5^{\circ}, 23.9^{\circ}, 26.2^{\circ}$ and $29.2^{\circ} 2 \theta$. As expected, CS did not exhibit any diffraction peak because of its amorphous nature. All ACV/CS freeze-dried formulations reflected similar diffraction patterns, where ACV characteristic peaks were detected with less intensity. This indicates that the freeze-drying process did not modify the crystalline structure of ACV, although peaks' intensity was clearly affected which could be due to crystallite size. Diffraction patterns of the formulations from L1 to L5 showed less intensity in ACV characteristic peaks due to the lower proportion of ACV compared to the X-ray diffraction patterns of systems with a higher amount of ACV (L6 $\rightarrow$ L10). Furthermore, slight displacements on ACV characteristic peaks were detected on some diffraction patterns (from $26.2^{\circ}$ to $26.3^{\circ} 2 \theta$; from $29.2^{\circ}$ to $29.3^{\circ} 2 \theta$ and from $23.9^{\circ}$ to $24.0^{\circ} 2 \theta$ ). It can be related to some modifications in the crystallite size during the lyophilization process. However, results suggest that there are no interactions between ACV and CS within systems in the studied ratios. 
Figure 1. X-Ray powder diffraction patterns of ACV (a); CS (b); and systems L1 (0.5:1 w/w ACV:CS) (c); L2 (0.5:2 w/w ACV:CS) (d); L3 (0.5:3 w/w ACV:CS) (e); L4 (0.5:4 w/w ACV:CS) (f); L5 (0.5:5 w/w ACV:CS) (g); L6 (2:1 w/w ACV:CS) (h); L7 (2:2 w/w ACV:CS) (i); L8 (2:3 w/w ACV:CS) (j); L9 (2:4 w/w ACV:CS) (k); and L10 (2:5 w/w $\mathrm{ACV}: \mathrm{CS})(\mathbf{l})$; in solid state.

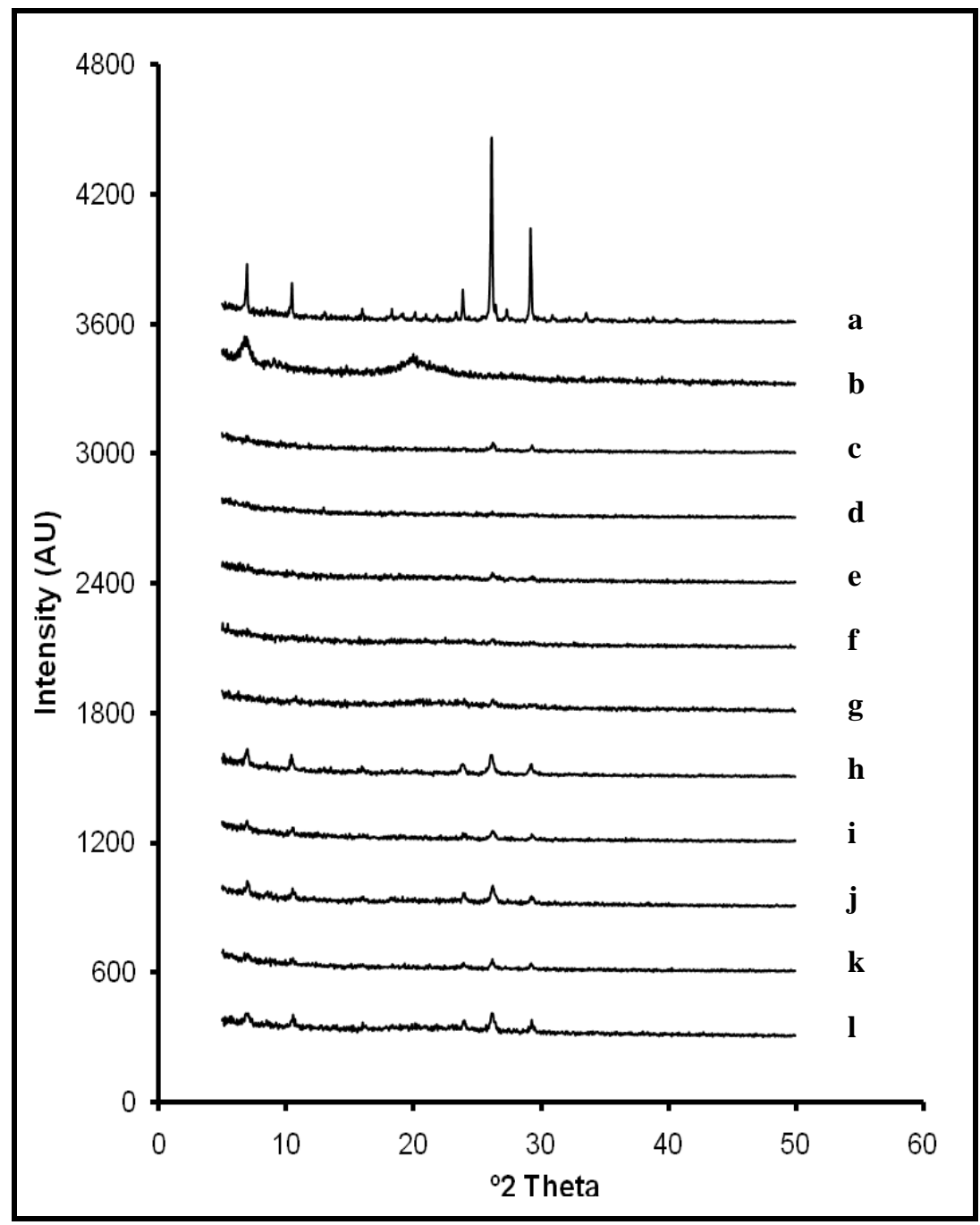

\subsection{Swelling Test}

Figure 2 shows the swelling ratios of systems in $\mathrm{HCl}$ medium $(\mathrm{pH}$ 1) (A) and in progressive $\mathrm{pH}$ medium (B), where each positive swelling ratio value indicates that, at this time, the swollen system weight was higher than the dry system weight $(\mathrm{t}=0)$. On the other hand, each negative swelling ratio value indicated that the weight of the swollen system was lower than the weight of the dry system $(t=0)$. When $t=0$, the swelling ratio value is 0 in all systems, due to the application of the equation shown in the experimental section.

The same two phase swelling behavior was observed in all the systems. The first phase of imbibition corresponds to a process of weighing gain, followed by a second phase of CS dissolution, resulting in a reduction of weight. In vitro swelling behavior has demonstrated to be influenced by the $\mathrm{ACV} / \mathrm{CS}$ ratios and medium nature. The maximum swelling ratios of every system and the time required for obtaining these values in both media are included in Table 2. 
Figure 2. Swelling ratio of freeze-dried systems B1 (1\% CS), B2 (2\% CS), B3 (3\% CS), B4 (4\% CS), B5 (5\% CS), L1 (0.5:1 w/w ACV:CS), L2 (0.5:2 w/w ACV:CS), L3 (0.5:3 w/w ACV:CS), L4 (0.5:4 w/w ACV:CS), L5 (0.5:5 w/w ACV:CS), L6 (2:1 w/w ACV:CS), L7 (2:2 w/w ACV:CS), L8 (2:3 w/w ACV:CS), L9 (2:4 w/w ACV:CS) and L10 (2:5 w/w ACV:CS) in $0.1 \mathrm{M} \mathrm{HCl}$ medium $(\mathrm{pH} 1)(\mathbf{A})$ and in progressive $\mathrm{pH}$ medium $(\mathrm{pH} 1.5 \rightarrow \mathrm{pH} 4)(\mathbf{B})$.

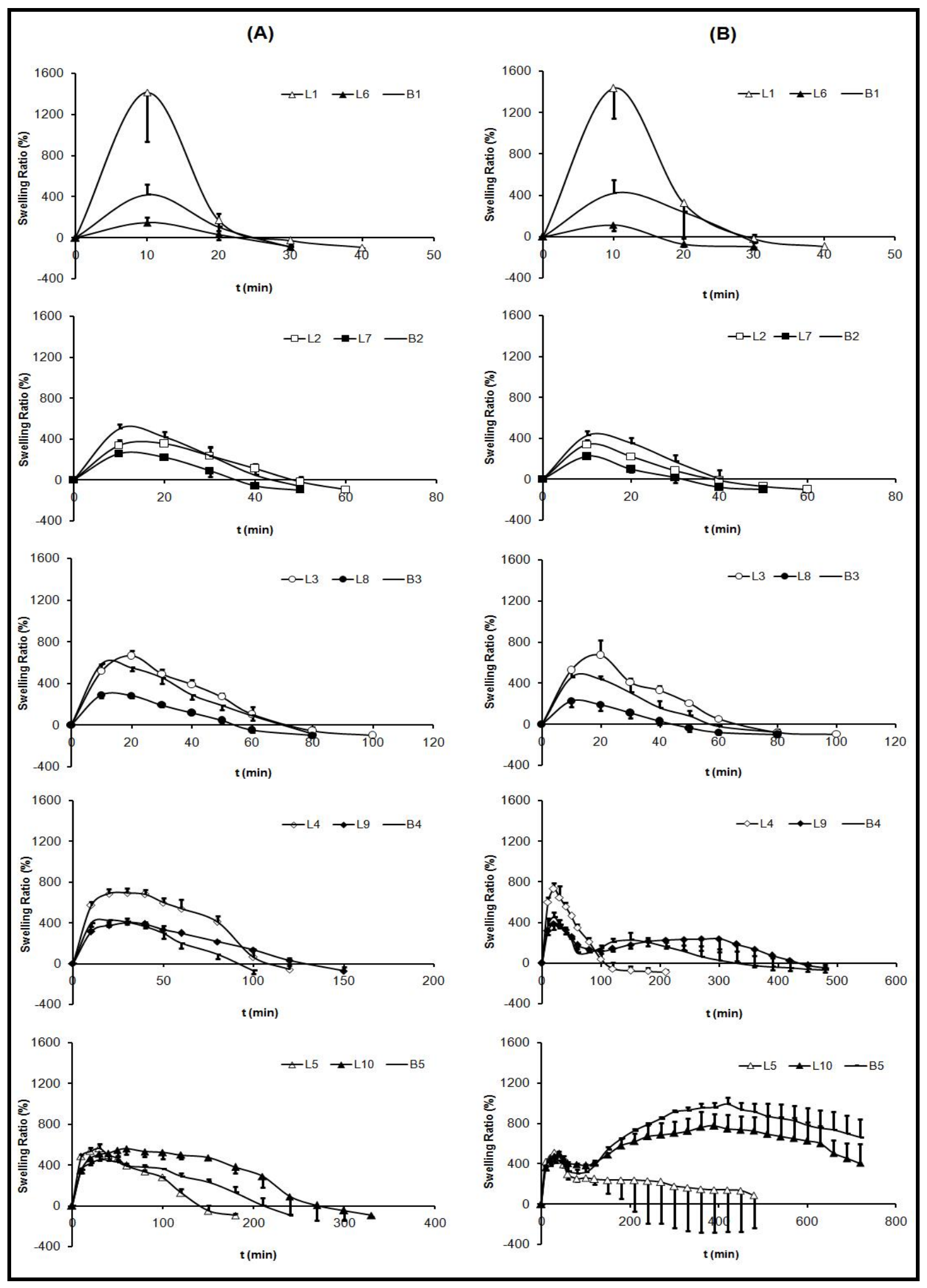


Influence of CS proportion: By studying blank systems (B), the characteristic CS swelling behavior has been determined. When increasing CS proportion within the system, lower swelling speed was obtained, lower amount of medium is imbibed, and therefore, a lower dissolution rate of CS was detected. Systems with lower proportions of polymer (B1, B2 and B3) showed clear differences between imbibition and dissolution phases. While, systems with high CS proportions (B4 and B5) displayed overlapped phases, because these systems were more compact and, as a consequence, they were able to maintain their shape for a prolonged time (Figures 3 and 4).

Table 2. Maximum swelling ratios (SR) and the time required for obtaining the values of freeze-dried systems: B1 (1\% CS), B2 (2\% CS), B3 (3\% CS), B4 (4\% CS), B5 (5\% CS), L1 (0.5:1 w/w ACV:CS), L2 (0.5:2 w/w ACV:CS), L3 (0.5:3 w/w ACV:CS), L4 (0.5:4 w/w ACV:CS), L5 (0.5:5 w/w ACV:CS), L6 (2:1 w/w ACV:CS), L7 (2:2 w/w ACV:CS), L8 (2:3 w/w ACV:CS), L9 (2:4 w/w ACV:CS) and L10 (2:5 w/w ACV:CS), in $0.1 \mathrm{M} \mathrm{HCl}$ medium ( $\mathrm{pH} 1)$ and in progressive $\mathrm{pH}$ medium $(\mathrm{pH} 1.5 \rightarrow \mathrm{pH} 4)$.

\begin{tabular}{ccccc}
\hline Formulations & \multicolumn{2}{c}{ 0.1 M HCl medium } & \multicolumn{2}{c}{ progressive pH medium } \\
\hline & Maximum SR (\%) & $\begin{array}{c}\text { T max } \\
\text { (min) }\end{array}$ & Maximum SR (\%) & $\begin{array}{c}\text { T max } \\
\text { (min) }\end{array}$ \\
\hline & & & & \\
B1 & $419.16 \pm 99.16$ & 10 & $420.41 \pm 125.25$ & 10 \\
B2 & $501.50 \pm 47.11$ & 10 & $428.44 \pm 45.24$ & 10 \\
B3 & $587.41 \pm 52.87$ & 10 & $457.51 \pm 75.75$ & 10 \\
B4 & $425.75 \pm 61.66$ & 20 & $453.11 \pm 43.88$ & 20 \\
B5 & $454.09 \pm 43.44$ & 30 & $992.05 \pm 63.13$ & 420 \\
L1 & $1413.92 \pm 476.74$ & 10 & $1433.65 \pm 289.10$ & 10 \\
L2 & $337.03 \pm 52.79$ & 10 & $338.01 \pm 48.10$ & 10 \\
L3 & $666.92 \pm 50.33$ & 20 & $673.52 \pm 146.14$ & 20 \\
L4 & $694.25 \pm 41.99$ & 30 & $730.73 \pm 58.05$ & 20 \\
L5 & $565.66 \pm 41.08$ & 30 & $511.96 \pm 63.49$ & 30 \\
L6 & $146.33 \pm 54.28$ & 10 & $111.92 \pm 58.52$ & 10 \\
L7 & $258.19 \pm 11.41$ & 10 & $225.02 \pm 24.29$ & 10 \\
L8 & $287.95 \pm 24.75$ & 10 & $227.24 \pm 59.78$ & 10 \\
L9 & $404.99 \pm 37.59$ & 30 & $387.88 \pm 59.51$ & 10 \\
L10 & $560.52 \pm 58.53$ & 60 & $777.00 \pm 114.73$ & 390 \\
\hline
\end{tabular}

Nevertheless, in progressive $\mathrm{pH}$ medium, four different phases can be observed: An imbibition process in $\mathrm{pH} 1.5$ medium; a CS dissolution process in $\mathrm{pH} \mathrm{1.5;} \mathrm{a} \mathrm{new} \mathrm{imbibition} \mathrm{process} \mathrm{when}$ achieving $\mathrm{pH} 4$ and finally; a slow $\mathrm{CS}$ dissolution in $\mathrm{pH} 4$ medium which demonstrated to last longer than 12 hours. Due to a rise in the $\mathrm{pH}$ of the medium, the CS dissolution was stopped and again systems absorbed medium. The aspect of the systems during swelling tests (Figures 3 and 4) was in accordance with the results obtained from swelling ratio (Figure 2). 
Figure 3. Evolution of freeze-dried formulations (F) B1 (1\% CS), B2 (2\% CS), B3 (3\% CS), B4 (4\% CS), B5 (5\% CS), L1 (0.5:1 w/w ACV:CS), L2 (0.5:2 w/w ACV:CS), L3 (0.5:3 w/w ACV:CS), L4 (0.5:4 w/w ACV:CS), L5 (0.5:5 w/w ACV:CS), L6 (2:1 w/w ACV:CS), L7 (2:2 w/w ACV:CS), L8 (2:3 w/w ACV:CS), L9 (2:4 w/w ACV:CS) and L10 $(2: 5 \mathrm{w} / \mathrm{w} \mathrm{ACV}: \mathrm{CS})$ for swelling study in $0.1 \mathrm{M} \mathrm{HCl}$ medium $(\mathrm{pH} 1)$.

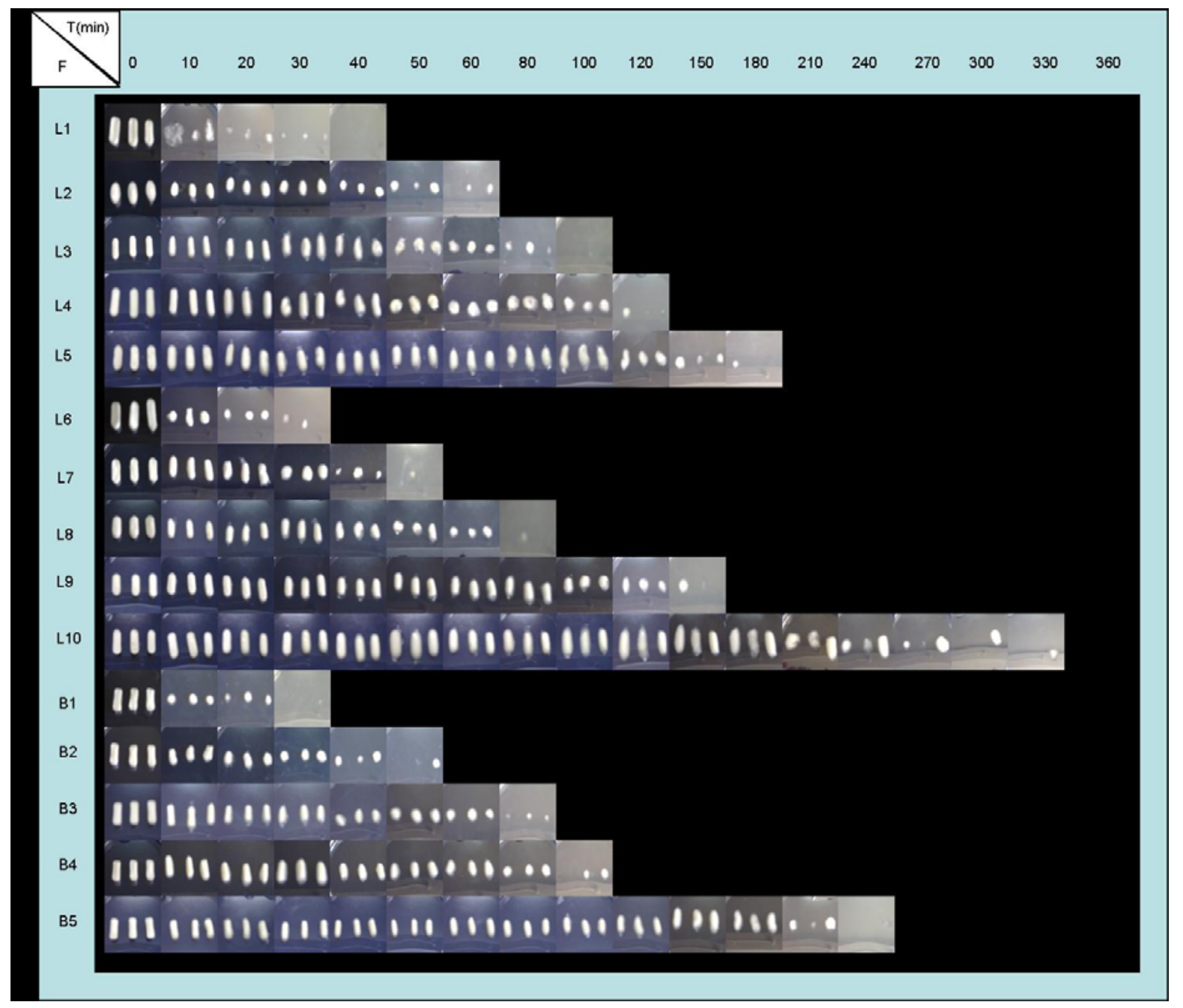

Influence of medium nature: Due to the pH-dependent CS solubility [1,4], some influence of medium nature was expected on swelling behavior. Unexpectedly however, systems with lower proportions of CS (1-3\%) exhibited similar swelling behavior in both media, due to completing imbibition and dissolution phases within one hour before progressive $\mathrm{pH}$ medium was reached. Instead, formulations with higher proportions of CS (B4, L4, L9, B5, L5 and L10) exhibited a prolonged swelling process (imbibitions phase), and in these cases, when increasing the $\mathrm{pH}$ medium (progressive $\mathrm{pH}$ medium), differences among swelling ratios were observed. The imbibition and CS dissolution phases could be clearly differed and occurred for less than six hours in $\mathrm{HCl}$ medium (pH 1).

Influence of ACV proportion: The incorporation of ACV within the systems modified the swelling behavior of $\mathrm{CS}$ due to ACV crystallites altering the linear structure of CS chains and, as a consequence, their ability to absorb aqueous media. A correlation between ACV/CS swelling behavior ratios can be defined. $0.5 \%$ ACV systems achieved higher weights in the imbibition phase than $2 \%$ ACV systems due to their capacity of water absorption. Regarding the CS dissolution phase, systems 
with low CS proportions (L1, L2, L3, L6, L7 and L8) showed similar behaviors, although in systems with $0.5 \% \mathrm{ACV}$ (L1, L2 and L3), the required time compared to the total CS dissolution is slightly higher, as can be seen in Figures 3 and 4. On the other hand, ACV systems with high amounts of CS (L4, L9, L5 and L10) exhibited a delay in the CS dissolution process, and also it was more prolonged in $2 \% \mathrm{ACV}$ systems (L9 and L10). The system that required longer to be dissolved was L10, which, even after 6 hours, remained undissolved.

Figure 4. Evolution of freeze-dried formulations (F) B1 (1\% CS), B2 (2\% CS), B3 (3\% $\mathrm{CS}), \mathrm{B} 4$ (4\% CS), B5 (5\% CS), L1 (0.5:1 w/w ACV:CS), L2 (0.5:2 w/w ACV:CS), L3 (0.5:3 w/w ACV:CS), L4 (0.5:4 w/w ACV:CS), L5 (0.5:5 w/w ACV:CS), L6 (2:1 w/w ACV:CS), L7 (2:2 w/w ACV:CS), L8 (2:3 w/w ACV:CS), L9 (2:4 w/w ACV:CS) and L10 $(2: 5 \mathrm{w} / \mathrm{w}$ ACV:CS) for swelling study, in progressive $\mathrm{pH}$ medium $(\mathrm{pH} 1.5 \rightarrow \mathrm{pH} 4)$.

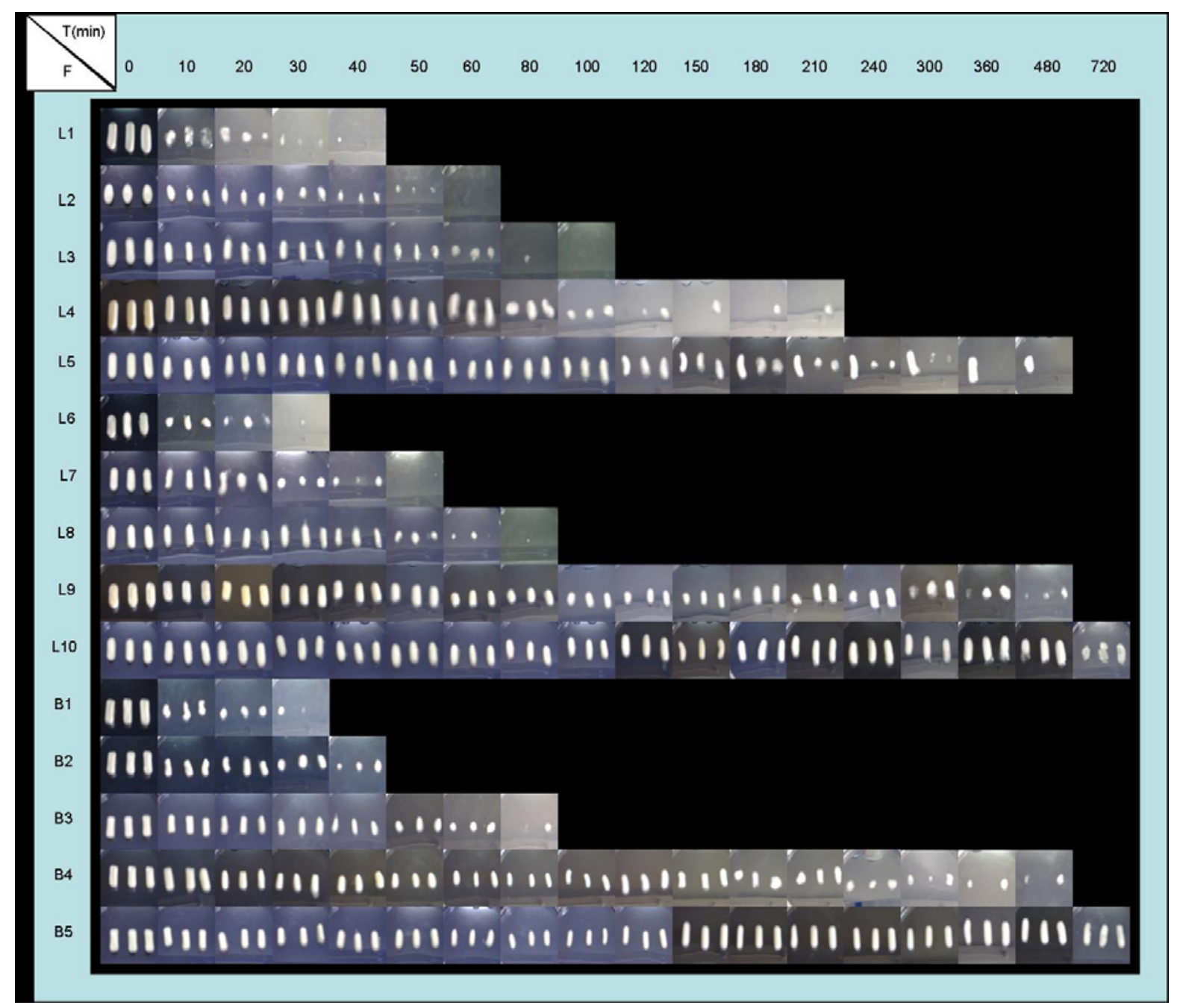

\subsection{Buoyancy Test}

All the studied lyophilized systems floated immediately upon contact with the release medium, showing no lag times in buoyancy of the systems. The low density of the systems could be a by-product of the freeze-drying process. 


\subsection{Dissolution Test}

Release profiles of ACV from freeze-dried formulations in $\mathrm{HCl}$ medium $(\mathrm{pH} 1)$ are represented in Figure 5 (A) and in progressive $\mathrm{pH}$ medium in Figure 5 (B). ACV release profiles from systems were influenced by the same factors as swelling behavior: CS and ACV ratios and medium nature.

Influence of CS proportion: ACV release profile was clearly influenced by CS proportion, as can be observed in Figure 5. As in the swelling phases, CS imbibition was the first step in the dissolution process. For this reason, an increase in the CS proportion produced more compact swollen systems and, therefore, more sustained profiles were obtained due to more time being required for dissolution and diffusion of ACV molecules within the system. On one hand, in $\mathrm{HCl}$ medium, at 40 minutes, the system L1 (low CS ratio) exhibited a dissolution profile where $100 \%$ of ACV was dissolved. While system L3 (medium CS ratio) required 120 minutes and system L5 (high CS ratio) required 330 minutes (Figure 5(A)).

On the other hand, in progressive $\mathrm{pH}$ medium, the influence of CS proportions in drug released was also detected (Figure 5(B)). The higher the CS ratio within the systems, the more sustained the releasing ACV profile. A conventional release profile was obtained from systems L1, L2, L6 and L7, while a sustained release profile was obtained from systems L4, L5, L9 and L10.

Influence of ACV proportion: Dissolution profiles obtained in $\mathrm{HCl}$ medium ( $\mathrm{pH}$ 1) from systems with the same amount of CS and different concentration of ACV (L1 and L6, L2 and L7, L3 and L8, L4 and L9 and L5 and L10) are similar, because ACV is soluble in this medium. Furthermore, the total drug dissolution from every system was only obtained when the freeze-dried formulations had completely lost their initial shape and aspect (Figure 3). However, at a progressive $\mathrm{pH}$ medium $(\mathrm{pH} 1.5 \rightarrow 4)$, the influence of ACV proportion over its selfsame dissolution was a little more remarkable. At 120 minutes the percentages of released ACV from the systems with an equal amount of CS and different amounts of ACV were: from L3, 96.7\% and from L8, 76.8\%; from L4, 68.1\% and from L9, 63.1\%; from L5, 64.6\% and from L10, 40.0\%. These different behaviors were due to the $\mathrm{pH}$ dependent ACV dissolution [52].

Influence of medium nature: The influence of dissolution medium composition in ACV release mechanism is clear in the systems with high proportions of CS and ACV, where the required time to obtain total drug dissolution was from 300 to 480 minutes (L5) and from 360 to 540 minutes (L10). Due to the fact that CS exhibits pH-dependency on its solubility $[1,4]$ and on imbibition phase, which was previously discussed in the swelling behavior section, ACV dissolution rate from the systems has shown to be influenced by the medium. Therefore, all the systems in $\mathrm{HCl}$ medium ( $\mathrm{pH} 1)$ exhibited total drug dissolution in less than six hours, while in progressive $\mathrm{pH}$ medium the duration of dissolution tests was prolonged to 12 hours in order to achieve complete drug dissolution of all formulations. During the first hour, similar dissolution rates were obtained in both media, due to the similarity of the $\mathrm{pH}$ values. However, after $\mathrm{pH}$ was changed from 1.5 to 4 , in the dissolution tests with progressive $\mathrm{pH}$ medium, the systems exhibited an ACV release more prolonged, being even more controlled in the systems with high amounts of ACV and CS. This could be related to the durability of the formulation in the progressive $\mathrm{pH}$ medium as previously explained in the four steps in the swelling behavior section. 
Figure 5. Dissolution profiles of freeze-dried systems (L) L1 (0.5:1 w/w ACV:CS), L2 (0.5:2 w/w ACV:CS), L3 (0.5:3 w/w ACV:CS), L4 (0.5:4 w/w ACV:CS), L5 (0.5:5 w/w ACV:CS), L6 (2:1 w/w ACV:CS), L7 (2:2 w/w ACV:CS), L8 (2:3 w/w ACV:CS), L9 (2:4 $\mathrm{w} / \mathrm{w}$ ACV:CS) and $\mathrm{L} 10(2: 5 \mathrm{w} / \mathrm{w} \mathrm{ACV}: \mathrm{CS})$ in $0.1 \mathrm{M} \mathrm{HCl} \mathrm{medium} \mathrm{(pH} \mathrm{1)} \mathrm{(A)} \mathrm{and} \mathrm{in}$ progressive $\mathrm{pH}$ medium $(\mathrm{pH} 1.5 \rightarrow \mathrm{pH} 4)(\mathbf{B})$.

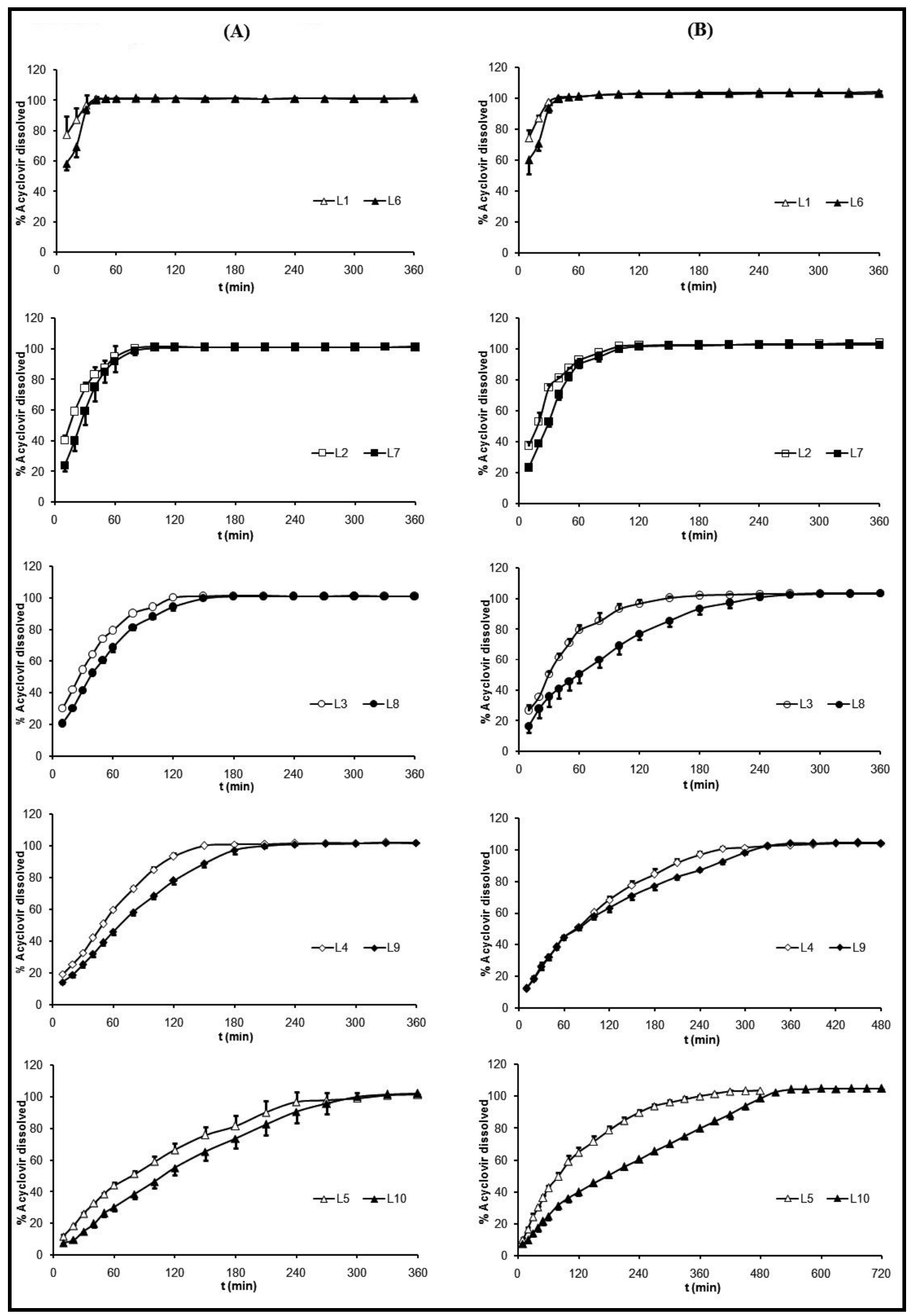




\section{Experimental Section}

\subsection{Materials}

Acyclovir (batch No. 14070620002) was a generous gift from CINFA (Pamplona, Spain). Chitosan (batch No. 8826900003) with a viscosity of $92.0 \mathrm{cSt}$ and a deacetylation degree of $97.0 \%$ was purchased from Guinama (Valencia, Spain). All other reagents were of analytical grade. PVC casts were a generous gift from Roche (Leganés, Madrid, Spain).

\subsection{Preparation of ACV/CS Freeze-Dried Formulations (L)}

$\mathrm{ACV}$, previously sieved through a mesh size of $100 \mu \mathrm{m}$, was dispersed into an aqueous solution of acetic acid ( $1 \% \mathrm{v} / \mathrm{v})$. Then, CS (previously sieved through a mesh size of $100 \mu \mathrm{m}$ ) was added to ACV suspension. The system was gently mixed with a magnetic stirrer until a homogeneous suspension was obtained. To prepare freeze-dried dosage forms, different suspensions of ACV/CS were dispensed into PVC casts and afterwards they were freeze-dried (Lio-Labor ${ }^{\circledR}$; Telstar, Barcelona, Spain) for 48 hours reaching a freezing temperature, a sublimation pressure and a sublimation temperature into a chamber of $-45{ }^{\circ} \mathrm{C}, 4.5410^{-4}$ atm and from -45 to $25{ }^{\circ} \mathrm{C}$, respectively. Blank freeze-dried formulations $(\mathrm{B} 1 \rightarrow \mathrm{B} 5)$ were also prepared, as described above, in order to compare them to the ACV/CS formulations in the characterization studies. The dimensions of systems were measured, and the results were $22.5 \pm 0.7 \mathrm{~mm}$ (length), $8.9 \pm 0.3 \mathrm{~mm}$ (width) and $6.1 \pm 0.2 \mathrm{~mm}$ (thickness) $(\mathrm{n}=15)$.

\subsection{Characterization of ACV/CS Lyophilized Formulations}

\subsubsection{X-ray diffraction analysis}

Powder X-ray diffraction patterns of raw materials and ACV/CS freeze-dried formulations were recorded by using an automated Philips ${ }^{\circledR}$ X'Pert X-ray diffractometer (Almelo, Netherlands). Samples were irradiated with monochromatized $\mathrm{Cu}-\mathrm{K} \alpha$ radiation and analyzed between $2 \theta$ angles of 5 and $40^{\circ}$. The current, the voltage and the time per step, were $55 \mathrm{~mA}, 40 \mathrm{mV}$ and $1 \mathrm{~s}$, respectively. A software package attached with the diffractometer was used to calculate the peak heights of all diffraction patterns (CAI, DRX, UCM). Powder X-ray diffraction patterns were measured in order to evaluate the crystalline/amorphous character of pure ingredients untreated and ACV/CS freeze-dried formulations.

\subsubsection{Swelling test}

Swelling ratio was evaluated as weight gain at a medium temperature of $37 \pm 0.1{ }^{\circ} \mathrm{C}$. As the gastric $\mathrm{pH}$ in fasted subjects is $1.1 \pm 0.15$ [53-55] and in fed state is 2.0 to 6.0 [56], the swelling, the buoyancy and the dissolution experiments were carried out in $0.1 \mathrm{M} \mathrm{HCl}(\mathrm{pH} 1)$ and in progressive $\mathrm{pH}$ medium $(1.5 \rightarrow 4)$. This progressive medium was composed of an aqueous mixture of $0.05 \mathrm{M}$ hydrochloric acid $37 \%, 0.05 \mathrm{M}$ ortho-phosphoric acid $85 \%$ and $0.05 \mathrm{M}$ acetic acid glacial with a final $\mathrm{pH}$ value of 1.5 , which was maintained during the first hour. After this hour, a sufficient quantity of $10 \mathrm{M} \mathrm{NaOH}$ was added until $\mathrm{pH}$ reached a value of 4.0, which was maintained until the test was finished.

The maximum duration of the tests was six hours in $0.1 \mathrm{M} \mathrm{HCl}$ medium $(\mathrm{pH} 1)$ and 12 hours in progressive $\mathrm{pH}$ medium. At specific time intervals, the samples were removed from test medium and 
were blotted with filter paper to absorb the excess of liquid on sample surface. At the same time, photographs were taken with a digital camera (Fujifilm ${ }^{\circledR}$ Finepix A345 (Tokyo, Japan) 4.1 Megapixels) to observe the appearance and evolution of all formulations in contact with the media. The swelling ratio (SR\%) of every sample was calculated according to the equation [57]:

$$
\mathrm{SR} \%=[(\mathrm{Ls}-\mathrm{Ld}) / \mathrm{Ld}] \times 100 \%
$$

where Ls and Ld were the weights of the swollen and dried samples respectively. All swelling tests were performed in triplicate.

\subsubsection{Buoyancy test}

All systems prepared were observed in order to determine their buoyancy ability, using the paddle method at a rotation speed of $100 \mathrm{rpm}$ with a Sotax ${ }^{\circledR}$ AT-7 dissolution apparatus (Basel, Switzerland) in $900 \mathrm{~mL}$ of either $0.1 \mathrm{M} \mathrm{HCl}(\mathrm{pH} 1)$ or progressive $\mathrm{pH}$ medium at $37^{\circ} \mathrm{C}$.

The maximum duration of assays was six hours in $0.1 \mathrm{M} \mathrm{HCl}$ medium $(\mathrm{pH} 1)$ and 12 hours in progressive $\mathrm{pH}$ medium. In every case, the samples performed contained $20 \mathrm{mg}$ of ACV. The position or location of the lyophilized systems during buoyancy tests was checked visually every hour. All buoyancy tests were performed in triplicate.

\subsubsection{Dissolution test}

A Sotax ${ }^{\circledR}$ AT-7 dissolution apparatus (Basel, Switzerland) with paddles was employed to carry out all tests. The paddle speed, volume of the dissolution medium and experimental temperature were $100 \mathrm{rpm}, 900 \mathrm{~mL}$ of either $0.1 \mathrm{M} \mathrm{HCl}(\mathrm{pH} 1)$ or progressive $\mathrm{pH}$ medium and $37 \pm 0.1^{\circ} \mathrm{C}$, respectively. A sample quantity of $20 \mathrm{mg}$ of ACV (previously sieved, size $<100 \mu \mathrm{m}$ ), or its equivalent amount from lyophilized systems, were used for all dissolution tests. The maximum duration of the tests was six hours in $0.1 \mathrm{M} \mathrm{HCl}$ medium ( $\mathrm{pH} 1$ ) and 12 hours in progressive $\mathrm{pH}$ medium. Samples were withdrawn at specific time intervals and filtered using Whatman ${ }^{\circledR}$ filter paper (type 42). The quantity of dissolved ACV was determined by UV spectroscopy at a wavelength of $255 \mathrm{~nm}(\mathrm{pH} 1$ and $\mathrm{pH} 1.5)$ and at $251.5 \mathrm{~nm}$ (pH 4), using in all cases a Beckman ${ }^{\circledR}$ DU-7 spectrophotometer (Brea, CA, U.S.). Three replicates of each dissolution test were carried out. The fact that there was no change in the $\lambda_{\max }$ of ACV because of the presence of CS had previously been checked.

\section{Conclusions}

The freeze-drying process enables ACV/CS floating systems to remain in gastric medium (fasted and fed states) until the total amount of ACV is released from the system. The amount of CS incorporated in the systems condition the swelling behavior and, consequently, the ACV release profile. These systems are capable of fast release formulations, releasing $100 \%$ of ACV within one hour (L1, L2, L6 and L7), or a more controlled release formulation which can release ACV over three hours or longer (L4, L5, L9 and L10). 


\section{Acknowledgements}

Roberto Ruiz Caro is a recipient of a predoctoral scholarship from the UCM (Spain) to do his PhD. This research was supported by an UCM project (PR27/05-13878). The authors would like to express their sincere gratitude to P. Ballesteros Papantonakis for her assistance and suggestions in the freezedrying process.

\section{References and Notes}

1. Illum, L. Chitosan and its use as a pharmaceutical excipient. Pharm. Res. 1998, 15, 1326-1331.

2. Skaugrud, O. Chitosan-New biopolymer for cosmetics and drugs. Drug Cosmetic Ind. 1991, 148, 24-29.

3. Ilango, R.; Jayakar, B.; Kavimani, S. Chitosan as a new pharmaceutical excipient. The East. Pharm. 1998, 41, 47-49.

4. Singla, A.K.; Chawla, M. Chitosan: some pharmaceutical and biological aspects-an update. $J$. Pharm. Pharmacol. 2001, 53, 1047-1067.

5. Burkatovskaya, M.; Castano, A.P.; Demidova-Rice, T.N.; Tegos, G.P.; Hamblin, M.R. Effect of chitosan acetate bandage on wound healing in infected and noninfected wounds in mice. Wound Repair Regen. 2008, 16, 425-431.

6. Chen, C.L.; Wang, Y.M.; Liu, C.F.; Wang, J.Y. The effect of water-soluble chitosan on macrophage activation and the attenuation of mite allergen-induced airway inflammation. Biomaterials 2008, 29, 2173-2182.

7. Seyfarth, F.; Schliemann, S.; Elsner, P.; Hipler, U.C. Antifungal effect of high- and lowmolecular-weight chitosan hydrochloride, carboxymethyl chitosan, chitosan oligosaccharide and $\mathrm{N}$-acetyl-D-glucosamine against Candida albicans, Candida krusei and Candida glabrata. Int. J. Pharm. 2008, 353, 139-148.

8. Gallaher, D.D.; Gallaher, C.M.; Mahrt, G.J.; Carr, T.P.; Hollingshead, C.H.; Hesslink, R., Jr.; Wise, J. A glucomannan and chitosan fiber supplement decreases plasma cholesterol and increases cholesterol excretion in overweight normocholesterolemic humans. J. Am. Coll. Nutr. 2002, 21, 428-433.

9. Chiang, M.T.; Yao, H.T.; Chen, H.C. Effect of dietary chitosans with different viscosity on plasma lipids and lipid peroxidation in rats fed on a diet enriched with cholesterol. Biosci. Biotechnol. Biochem. 2000, 64, 965-971.

10. Ubaidulla, U.; Khar, R.K.; Ahmad, F.J.; Sultana, Y.; Panda, A.K. Development and characterization of chitosan succinate microspheres for the improved oral bioavailability of insulin. J. Pharm. Sci. 2007, 96, 3010-3023.

11. Lueßen, H.L.; Lehr, C.M.; Rentel, C.O.; Noach, A.B.J.; de Boer, A.G.; Verhoef, J.C.; Junginger, H.E. Bioadhesive polymers for the peroral delivery of peptide drugs. J. Control. Release 1994, 29 , 329-338.

12. Lueßen, H.L.; de Leeuw, B.J.; Langemeÿer, M.W.; de Boer, A.G.; Verhoef, J.C.; Junginger, H.E. Mucoadhesive polymers in peroral peptide drug delivery. VI. Carbomer and chitosan improve the intestinal absorption of the peptide drug buserelin in vivo. Pharm. Res. 1996, 13, 1668-1672. 
13. Lueßen, H.L.; Rentel, C.O.; Kotzé, A.F.; Lehr, C.M.; de Boer, A.G.; Verhoef, J.C.; Junginger, H.E. Mucoadhesive polymers in peroral peptide drug delivery. IV. Polycarbophil and chitosan are potent enhancers of peptide transport across intestinal mucosae in vitro. J. Control. Release 1997, $45,15-23$.

14. Hassan, E.E.; Gallo, J.M. A simple rheological method for the in vitro assessment of mucinpolymer bioadhesive bond strength. Pharm. Res. 1990, 7, 491-495.

15. Kofuji, K.; Shibata, K.; Murata, Y.; Miyamoto, E.; Kawashima, S. Preparation and drug retention of biodegradable chitosan gel beads. Chem. Pharm. Bull. 1999, 47, 1494-1496.

16. Ganza-González, A.; Anguiano-Igea, S.; Otero-Espinar, F.J.; Blanco Méndez, J. Chitosan and chondroitin microspheres for oral-administration controlled release of metoclopramide. Eur. J. Pharm. Biopharm. 1999, 48, 149-155.

17. Shantha, K.L.; Harding, D.R.K. Preparation and in-vitro evaluation of poly[N-vinyl-2pyrrolidone-polyethylene glycol diacrylate]-chitosan interpolymeric $\mathrm{pH}$-responsive hydrogels for oral drug delivery. Int. J. Pharm. 2000, 207, 65-70.

18. Park, S.H.; Chun, M.K.; Choi, H.K. Preparation of an extended-release matrix tablet using chitosan/Carbopol interpolymer complex. Int. J. Pharm. 2008, 347, 39-44.

19. Nunthanid, J.; Laungtana-Anan, M.; Sriamornsak, P.; Limmatvapirat, S.; Puttipipatkhachorn, S.; Lim, L.Y.; Khor, E. Characterization of chitosan acetate as a binder for sustained release tablets. J. Control. Release 2004, 99, 15-26.

20. Zerrouk, N.; Mennini, N.; Maestrelli, F.; Chemtob, C.; Mura, P. Comparison of the effect of chitosan and polyvinylpyrrolidone on dissolution properties and analgesic effect of naproxen. Eur. J. Pharm. Biopharm. 2004, 57, 93-99.

21. Park, K.M.; Bae, J.W.; Joung, Y.K.; Shin, J.W.; Park, K.D. Nanoaggregate of thermosensitive chitosan-Pluronic for sustained release of hydrophobic drug. Colloids Surf. B. 2008, 63, 1-6.

22. Lim Soo, P.; Cho, J.; Grant, J.; Ho, E.; Piquette-Miller, M.; Allen, C. Drug release mechanism of paclitaxel from a chitosan-lipid implant system: Effect of swelling, degradation and morphology. Eur. J. Pharm. Biopharm. 2008, 69, 149-157.

23. McConnell, E.L.; Murdan, S.; Basit, A.W. An Investigation into the Digestion of Chitosan (Noncrosslinked and Crosslinked) by Human Colonic Bacteria. J. Pharm. Sci. 2008, 97, 3820-3829.

24. Bonferoni, M.C.; Sandri, G.; Rossi, S.; Ferrari, F.; Gibin, S.; Caramella, C. Chitosan citrate as multifunctional polymer for vaginal delivery. Evaluation of penetration enhancement and peptidase inhibition properties. Eur. J. Pharm. Sci. 2008, 33, 166-176.

25. Strauss, E.G.; Strauss, J.H. Viruses and Human Disease, 1st ed.; Academic Press: San Diego, CA, USA, 2002.

26. Whitley, R.J. Herpes simplex virus. In Fields virology, 4th ed.; Knipe, D.M.; Howley, P.M., Eds.; Lippincott, Williams and Wilkins: Philadelphia, PA, USA, 2001; pp. 2461-2511.

27. Whitley, R.J.; Roizman, B. Herpes simplex virus infections. Lancet 2001, 357, 1513-1518.

28. Spruance, S.L.; Overall, J.C., Jr.; Kern, E.R.; Krueger, G.G.; Pliam, V.; Miller, W. The natural history of recurrent herpes simplex labialis: implications for antiviral therapy. N. Engl. J. Med. 1977, 297, 69-75. 
29. Norval, M.; el-Ghorr, A.A. UV radiation and mouse models of herpes simplex virus infection. Photochem. Photobiol. 1996, 64, 242-245.

30. Erlich, K.S.; Mills, J.; Chatis, P.; Mertz, G.J.; Busch, D.F.; Follansbee, S.E.; Grant, R.M.; Crumpacker, C.S. Acyclovir-resistant herpes simplex virus infections in patients with the acquired immunodeficiency syndrome. N. Engl. J. Med. 1989, 320, 293-296.

31. Corey, L.; Spear, P.G. Infections with herpes simplex viruses (1). N. Engl. J. Med. 1986, 314, 686-691.

32. Corey, L.; Spear, P.G. Infections with herpes simplex viruses (2). N. Engl. J. Med. 1986, 314, 749-757.

33. Erlich, K.S. Management of herpes simplex and varicella-zoster virus infections. West. J. Med. 1997, 166, 211-215.

34. Fujioka, Y.; Mizuno, N.; Morita, E.; Motozono, H.; Takahashi, K.; Yamanaka, Y.; Shinkuma, D. Effect of age on the gastrointestinal absorption of acyclovir in rats. J. Pharm. Pharmacol. 1991, 43, 465-469.

35. Meadows, K.C.; Dressman, J.B. Mechanism of acyclovir uptake in rat jejunum. Pharm. Res. 1990, 7, 299-303.

36. Vergin, H.; Kikuta, C.; Mascher, H.; Metz, R. Pharmacokinetics and bioavailability of different formulations of acyclovir. Arzneimittelforschung 1995, 45, 508-515.

37. de Miranda, P.; Krasny, H.C.; Page, D.A.; Elion, C.B. Species differences in the disposition of acyclovir. Am. J. Med. 1982, 73, 31-35.

38. Collins, P.; Bauer, D.J. Relative potencies of anti-herpes compounds. Ann. N. Y. Acad. Sci. 1977, 284, 49-59.

39. Krenitsky, T.A.; Hall, W.W.; de Miranda, P.; Beauchamp, L.M.; Schaeffer, H.J.; Whiteman, P. 6Deoxy acyclovir: a xanthine oxidase-activated prodrug of acyclovir. Proc. Natl. Acad. Sci. USA 1984, 81, 3209-3213.

40. Lycke, J.; Malmeström, C.; Ståhle, L. Acyclovir levels in serum and cerebrospinal fluid after oral administration of valacyclovir. Antimicrob. Agents Chemother. 2003, 47, 2438-2441.

41. Luengo, J.; Aránguiz, T.; Sepulveda, J. Preliminary pharmacokinetic study to different preparations of acyclovir with $\beta$-cyclodextrin. J. Pharm. Sci. 2002, 91, 2593-2598.

42. Attia, I.A.; El-Gizawy, S.A; Fouda, M.A.; Donia, A.M. Influence of a niosomal formulation on the oral bioavailability of acyclovir in rabbits. AAPS PharmSciTech. 2007, 8, E106.

43. Lewis, L.D.; Fowle, A.S.; Bittiner, S.B.; Bye, A.; Isaacs, P.E. Human gastrointestinal absorption of acyclovir from tablet duodenal infusion and sipped solution. Br. J. Clin. Pharmacol. 1986, 21, 459-462.

44. Özdemir, N.; Ordu, S.; Özkan, Y. Studies of floating dosage forms of furosemide: in vitro and in vivo evaluations of bilayer tablet formulations. Drug Dev. Ind. Pharm. 2000, 26, 857-866.

45. Hoffman, A.; Stepensky, D.; Lavy, E.; Eyal, S.; Klausner, E.; Friedman, M. Pharmacokinetic and pharmacodynamic aspects of gastroretentive dosage forms. Int. J. Pharm. 2004, 277, 141-153.

46. Gröning, R.; Cloer, C.; Georgarakis, M.; Müller, R.S. Compressed collagen sponges as gastroretentive dosage forms: in vitro and in vivo studies. Eur. J. Pharm. Sci. 2007, 30, 1-6.

47. Sheth, P.R.; Tossounian, J.L. Novel sustained release tablet formulations. U.S. Patent 4,167,558, 1979. 
48. Junyaprasert, V.B.; Pornsuwannapha, S. Floating properties and release characteristics of hollow microspheres of acyclovir. Drug Deliv. 2008, 15, 331-341.

49. Gröning, R.; Berntgen, M.; Georgarakis, M. Acyclovir serum concentrations following peroral administration of magnetic depot tablets and the influence of extracorporal magnets to control gastrointestinal transit. Eur. J. Pharm. Biopharm. 1998, 46, 285-291.

50. Whitehead, L.; Fell, J.T.; Collett, J.H.; Sharma, H.L.; Smith, A.M. Floating dosage forms: an in vivo study demonstrating prolonged gastric retention. J. Control. Release 1998, 55, 3-12.

51. Talukder, R.; Fassihi, R. Gastroretentive delivery systems: hollow beads. Drug Dev. Ind. Pharm. 2004, 30, 405-412.

52. US FDA. Waiver of in vivo bioavailability and bioequivalence studies for immediate-release solid dosage forms based on a biopharmaceutics classification system. 2000. Available online: http://www.fda.gov/downloads/Drugs/GuidanceComplianceRegulatoryInformation/Guidances/uc m070246.pdf (accessed on 1 September 2010).

53. Dressman, J.B.; Berardi, R.R.; Dermentzoglou, L.C.; Russell, T.L.; Schmaltz, S.P.; Barnett, J.L.; Jarvenpaa, K.M. Upper gastrointestinal (GI) pH in young, healthy men and women. Pharm. Res. 1990, 7, 756-761.

54. Russell, T.L.; Berardi, R.R.; Barnett, J.L.; Dermentzoglou, L.C.; Jarvenpaa, K.M.; Schmaltz, S.P.; Dressman, J.B. Upper gastrointestinal pH in seventy-nine healthy, elderly, North American men and women. Pharm. Res. 1993, 10, 187-196.

55. Lui, C.Y.; Amidon, G.L.; Berardi, R.R.; Fleisher, D.; Youngberg, C.; Dressman, J.B. Comparison of gastrointestinal $\mathrm{pH}$ in dogs and humans: implications on the use of the beagle dog as a model for oral absorption in humans. J. Pharm. Sci. 1986, 75, 271-274.

56. Arora, S.; Ali, J.; Ahuja, A.; Khar, R.K.; Baboota, S. Floating drug delivery systems: a review. AAPS PharmSciTech 2005, 6, E372-E390.

57. Haupt, S.; Zioni, T.; Gati, I.; Kleinstern, J.; Rubinstein, A. Luminal delivery and dosing considerations of local celecoxib administration to colorectal cancer. Eur. J. Pharm. Sci. 2006, 28, 204-211.

(C) 2010 by the authors; licensee MDPI, Basel, Switzerland. This article is an open access article distributed under the terms and conditions of the Creative Commons Attribution license (http://creativecommons.org/licenses/by/3.0/). 